\title{
STRUCTURE-BASED MULTITARGETED MOLECULAR DOCKING ANALYSIS OF PYRAZOLE- CONDENSED HETEROCYCLICS AGAINST LUNG CANCER
}

\author{
JAINEY P. JAMES ${ }^{1}{ }^{*}$, AISWARYA T. C. ${ }^{1}$, SNEH PRIYA ${ }^{1}$, DIVYA JYOTHI ${ }^{1}$, SHESHAGIRI R. DIXIT ${ }^{2}$
}

${ }^{1}$ Nitte (Deemed to be University), NGSM Institute of Pharmaceutical Sciences (NGSMIPS), Deralakatte, Mangaluru 575018, Karnataka, India, 2Department of Pharmaceutical Chemistry, JSS College of Pharmacy, JSS Academy of Higher Education and Research, Mysuru $\mathbf{5 7 0 0 1 5}$, Karnataka, India

Email: jaineyjames@nitte.edu.in

Received: 02 Aug 2021, Revised and Accepted: 03 Sep 2021

\section{ABSTRACT}

Objective: The significant drawbacks of chemotherapy are that it destroys healthy cells, resulting in adverse effects. Hence, there is a need to adopt new techniques to develop cancer-specific chemicals that target the molecular pathways in a non-toxic fashion. This study aims to screen pyrazolecondensed heterocyclics for their anticancer activities and analyse their enzyme inhibitory potentials EGFR, ALK, VEGFR and TNKS receptors.

Methods: The structures of the compounds were confirmed by IR, NMR and Mass spectral studies. The in silico techniques applied in this study were molecular docking and pharmacophore modeling to analyse the protein-ligand interactions, as they have a significant role in drug discovery. Drug-likeness properties were assessed by the Lipinski rule of five and ADMET properties. Anticancer activity was performed by in vitro MTT assay on lung cancer cell lines.

Results: The results confirm that all the synthesised pyrazole derivatives interacted well with the selected targets showing docking scores above- 5 $\mathrm{kcal} / \mathrm{mol}$. Pyrazole $2 \mathrm{e}$ interacted well with all the four lung cancer targets with its stable binding mode and was found to be potent as per the in vitro reports, followed by compounds $3 \mathrm{~d}$ and $2 \mathrm{~d}$. Pharmacophore modeling exposed the responsible features responsible for the anticancer action. ADMET properties reported that all the compounds were found to have properties within the standard limit. The activity spectra of the pyrazoles predicted that pyrazolopyridines (2a-2e) are more effective against specific receptors such as EGFR, ALK and Tankyrase.

Conclusion: Thus, this study suggests that the synthesised pyrazole derivatives can be further investigated to validate their enzyme inhibitory potentials by in vivo studies.

Keywords: Lung cancer, Pyrazolopyrimidines, Pyrazolopyridines, Molecular docking, Pharmacophore modeling, Anticancer activities

(C) 2021 The Authors. Published by Innovare Academic Sciences Pvt Ltd. This is an open access article under the CC BY license (https://creativecommons.org/licenses/by/4.0/) DOI: https://dx.doi.org/10.22159/ijap.2021v13i6.42801. Journal homepage: https://innovareacademics.in/journals/index.php/ijap

\section{INTRODUCTION}

Lung cancer is one of the leading causes of cancer mortality in men and women, [1, 2] responsible for 1.6 million deaths. Non-small-cell lung cancers (NSCLCs), including large-cell carcinoma, adenocarcinoma, and squamous cell carcinoma, contribute approximately $80-85 \%$ of lung cancer.

The major shortcoming of lung cancer chemotherapy is that it causes damages to normal cells, causing surplus adverse effects. Therefore targeted therapies [3] are needed to target only cancer cells, avoiding injuries to the healthy cells. One of the novel methods adopted in lung cancer therapy is developing cancer-specific compounds that can attack the molecular signalling pathways, thus creating non-toxic substances. The significant targets of paramount importance for lung cancers are EGFR (Epidermal growth factor receptor) [4, 5] ALK (Anaplastic lymphoma tyrosine kinase) [6, 7] BRAF (v-raf murine sarcoma viral oncogene homolog B1) [8, 9] VEGFR receptors (Vascular endothelial growth factor) [10,11], and Wnt signalling pathway [12].

The EGFR receptor is recognised as a significant anticancer target. It belongs to the ErbB (epidermal growth factor) receptor tyrosine kinase family and is expressed at high levels on the surface of some cancer cells. The inhibition of EGFR plays a crucial role in angiogenesis, tumour suppression, and metastasis [13].

In anaplastic non-Hodgkin's lymphoma, the ALK gene was first described as a driver mutation. Dysregulated ALK expression is now an identified driver mutation in nearly twenty different human malignancies. The dysregulated ALK expression is now recognised as the driver mutation, including $4-9 \%$ of NSCLC [14].

One of the critical mediators promoting the angiogenesis process is VEGFR, as it has a prominent role in maintaining the vascular supply within the tumour. Its increased levels are a confirmatory factor in diverse human cancers, including NSCLC [15]. The Wnt signalling pathway is another potential target for lung cancer. Effective pharmacological inhibitors of the Wnt pathway have only recently become available. The tankyrase (TNKS), a poly-ADP-ribose polymerase (PARP) enzyme, was the critical mediator of Wnt signalling by the screens for small molecular antagonists of the Wnt pathway. Hence, using the targets mentioned above as partial agonists/antagonists can show promising treatment strategies [16].

The approved therapeutic EGFR inhibitors are gefitinib, erlotinib, afatinib, osimertinib, dacomitinib [17], and ALK inhibitors crizotinib, alectinib, brigatinib, lorlatinib [18], VEGFR inhibitors are axitinib, bevacizumab, sorafenib [19]etc.

Nitrogen-containing heterocycle-pyrazole has a vital role in the development of cancer therapies. The anticancer activity of these compounds is by the inhibition of different types of proteins, receptors and enzymes, which has a crucial role in cell division. Condensed pyrazole rings such as pyrazolopyrimidines, pyrazolopyridines and pyranopyrazole are known for their anticancer properties [20], and the available drugs with these core moieties are depicted in fig. 1.

An extension of previous works on pyrazole scaffolds [21, 22] and in silico studies $[23,24]$, we have performed an analysis to screen the inhibitory potency of synthesized pyrazole fused derivatives on various targets EGFR, ALK, VEGFR and TNKS by employing molecular docking and pharmacophore modelling techniques.

\section{MATERIALS AND METHODS}

Most of the chemicals were purchased from Sigma Aldrich, and further purification was not required. Melting points was determined by the capillary method and were uncorrected. Shimadzu Perkin Ekmer 8201 
Pc IR Spectrometer used in recording IR spectra ( $\mathrm{KBr}$ pellets), and frequencies are expressed in $\mathrm{cm}^{-1}$. Bruker Avance II, 400 NMR spectrometer, recorded NMR spectra and Shimadzu LCMS 8030, Japan Mass spectrometer recorded mass spectra.

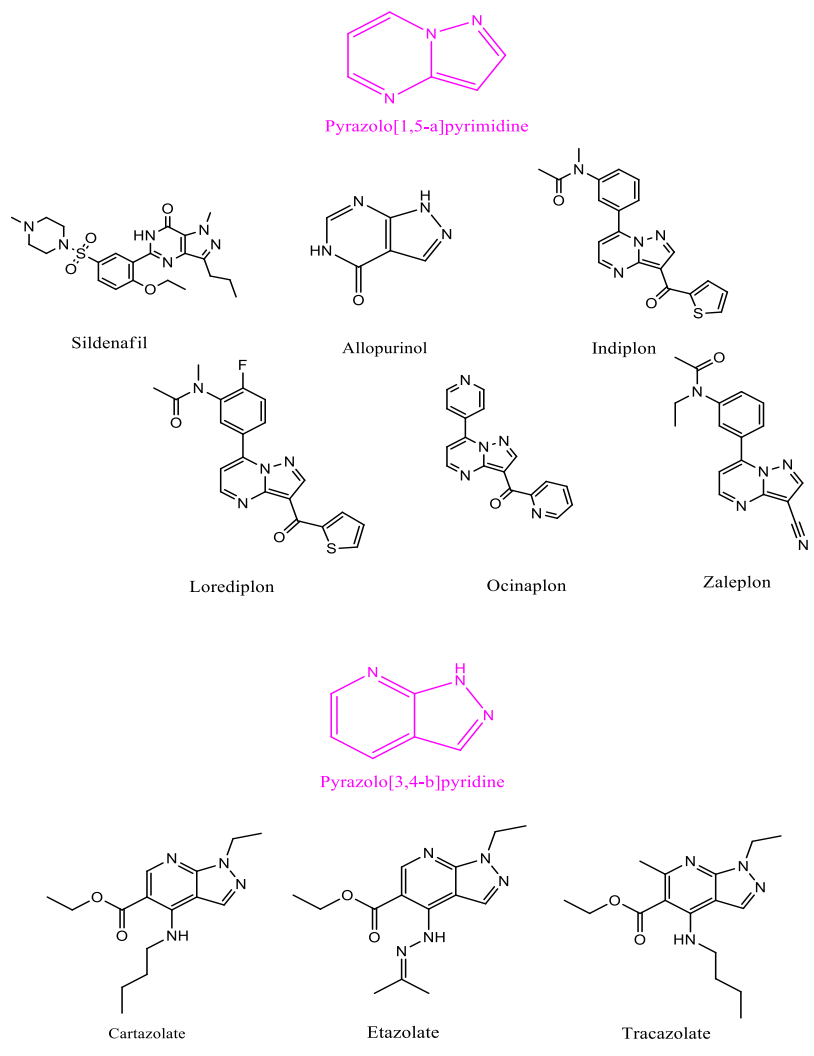

Fig. 1: Available drugs with pyrazolopyrimidines and pyrazolopyridines moiety

\section{Preparation of pyrazolopyrimidines (2a-2e) and pyrazolo- pyridines (3a-3e)}

A solution of 0.01 mole of malonitrile/diethyl malonate and different pyrazole carbonitrile derivatives 1 ( 0.01 mole) was prepared in sodium ethoxide and ethanol, which was refluxed for eight hours. The solution was concentrated, and the obtained residue was filtered, washed with ice-cold water [25].

\section{Modeling platform}

In silico analysis was carried out on Maestro 11.9 (Schrödinger, 2019-4) [26]. This software package is programmed on DELL Inc.27" workstation machine running on Intel Core i7-7700 CPU@ 3.60 GHz $\mathrm{x} 8$, a processor with 8GB RAM and 1000 GB hard disk with Linuxx86_64 as the operating system.

\section{Molecular docking and binding free energy calculation}

Based on the literature, the selected targets for lung cancers are EGFR, ALK, VEGFR and tankyrase and their crystal structures EGFR (PDB ID: 4WKQ) [27], ALK (PDB ID: 4Z55) [28], VEGFR (PDB ID: 4AG8) [29], TNKS (PDB ID: 4W5S) [30] were availed from the protein data bank. The downloaded proteins were minimised by Protein Preparation Wizard, using the OPLS-2005 force field of Schrodinger software. The designed fused-pyrazoles were prepared by LigPrep application (Schrödinger, 2019-4) [26] and were used for docking. The minimized protein was employed to generate the grid, and the grid box was developed by applying default parameters. Glide-XP (extra precision) [31] was used for molecular docking computations. The binding free energy MMGBSA (Molecular Mechanics, Generalized Born Model and Solvent Accessibility) dGbind ( $\mathrm{kcal} / \mathrm{mol})$, between the receptor and ligands, were calculated by the Prime module (Schrödinger, 2019-4) [26]. The docking scores and the 2D and 3D conformations were generated to analyse further the affinities and binding interactions of the selected ten fused-pyrazole molecules.
The docking procedure was confirmed by redocking the co-crystal ligand of the proteins into the binding sites, respectively. The docking poses of the co-crystals in XP method and experimental conditions were compared and found to be similar with RMSD, thus validating the docking results.

\section{Pharmacophore modeling}

Pharmacophore modeling was performed by Phase tool (Schrödinger, 2019-4) [26]. In this model, six pyrazoles were considered active ( $\geq 69 \%$ ), and four were inactive based on their percent inhibition on lung cancer cells. Common pharmacophore hypotheses $(\mathrm{CPH})$ were searched, and the best $\mathrm{CPH}$ was selected based on the survival score until at least one hypothesis was found and scored successfully. Pharmacophore-matching tolerance was set to $2 \mathrm{~A}^{\circ}$.

Drug-likeness, ADMET property and prediction of activity spectral studies

The compounds were screened for drug-likeness properties by checking with the Lipinski Rule of five [32] and ADMET (Absorption, Distribution, Metabolism, Excretion and Toxicity) property prediction by the QikProp tool [26]. The features considered for ADMET studies are the following: QPlogHERG, QPPCaco Caco-2 cell permeability, QPlogKhsa, Percent Human Oral Absorption. Further, to validate them as appropriate drug candidates, an online tool, prediction of activity spectra for substances (PASS), was used, which evaluate the biological activity based on their structural data [33]. This tool gives the values for the probability of activity $(\mathrm{Pa})$ and inactivity (Pi) by comparing more than 300 pharmacological effects and biochemical mechanisms of compounds.

\section{In vitro anticancer study by MTT assay}

We procured A-549 (Human small-cell lung carcinoma) cell culture from National Centre for Cell Sciences (NCCS), Pune, India. Ten 
compounds were incubated with different concentrations $(25,50$ $100,200 \mu \mathrm{M}$ ) to screen the cytotoxic activity of the compounds against human small-cell lung carcinoma (A-549). The cell viability was then determined by the MTT (3-(4,5-dimethylthiazol-2-yl)-2,5diphenyltetrazolium bromide) assay after $24 \mathrm{~h}$ of incubation. Percent inhibition was calculated from the absorbance as $\%$ growth inhibition [34].

\section{RESULTS}

\section{Chemistry}

The fused pyrazole derivatives were synthesized from substituted aminopyrazoles cyclising with malononitrile and diethyl malonate to yield pyrazolopyridines and pyrazolopyrimidines. IR, NMR and mass spectroscopic techniques were used to confirm the structures (table 1).

Table 1: Structure and spectral data of pyrazole derivatives

\begin{tabular}{|c|c|c|c|c|c|}
\hline S. No. & Compound code & Structure & IR (KBr, $\left.\mathrm{cm}^{-1}\right)$ & 1H NMR (400 MHz, DMSO, $\delta / p p m$ & LC-MS (m/z) \\
\hline 1. & $2 a$ & & $\begin{array}{l}1625.78(\mathrm{C}=\mathrm{N}), \\
1583.61(\mathrm{C}=\mathrm{C}), \\
3463.56 \text { and } 3296.45 \\
\left(\mathrm{NH}_{2}\right), 2213.54(\mathrm{CN})\end{array}$ & $\begin{array}{l}3.67\left(\mathrm{~s}, 2 \mathrm{H}, \mathrm{CH}_{2}\right), 7.74\left(\mathrm{~s}, 2 \mathrm{H}, \mathrm{NH}_{2}\right) \\
8.19(\mathrm{~s}, 1 \mathrm{H}, \mathrm{CH}), 7.51-7.60(\mathrm{~m}, 5 \mathrm{H}, \mathrm{Ar}- \\
\mathrm{H})\end{array}$ & $\left(\mathrm{M}^{+}\right) 250$ \\
\hline 2. & $2 b$ & & $\begin{array}{l}1666.38(\mathrm{C}=\mathrm{N}), \\
1592.32(\mathrm{C}=\mathrm{C}), \\
3423.76 \text { and } 3265.45 \\
\left(\mathrm{NH}_{2}\right), 2219.34(\mathrm{CN}), \\
767.97(\mathrm{C}-\mathrm{Cl})\end{array}$ & $\begin{array}{l}3.12\left(\mathrm{~s}, 2 \mathrm{H}, \mathrm{CH}_{2}\right), 7.68\left(\mathrm{~s}, 2 \mathrm{H}, \mathrm{NH}_{2}\right) \\
8.33(\mathrm{~s}, 1 \mathrm{H}, \mathrm{CH}), 7.52-7.61(\mathrm{~m}, 4 \mathrm{H}, \mathrm{Ar}- \\
\mathrm{H})\end{array}$ & $\left(\mathrm{M}^{+}\right) 284$ \\
\hline 3. & $2 c$ & & $\begin{array}{l}1635.89(\mathrm{C}=\mathrm{N}), \\
1593.82(\mathrm{C}=\mathrm{C}), \\
3421.45 \text { and } 3288.32 \\
\left(\mathrm{NH}_{2}\right), 2214.43(\mathrm{CN}), \\
786.23(\mathrm{C}-\mathrm{Cl})\end{array}$ & $\begin{array}{l}3.31\left(\mathrm{~s}, 2 \mathrm{H}, \mathrm{CH}_{2}\right), 7.58\left(\mathrm{~s}, 2 \mathrm{H}, \mathrm{NH}_{2}\right) \\
8.35(\mathrm{~s}, 1 \mathrm{H}, \mathrm{CH}), 7.53-7.60(\mathrm{~m}, 4 \mathrm{H}, \mathrm{Ar}- \\
\mathrm{H})\end{array}$ & $\left(\mathrm{M}^{+}\right) 284$ \\
\hline 4. & $2 \mathrm{~d}$ & & $\begin{array}{l}1646.32(\mathrm{C}=\mathrm{N}), \\
1594.69(\mathrm{C}=\mathrm{C}), \\
3408.08 \text { and } 3269.67 \\
\left(\mathrm{NH}_{2}\right), 2245.76(\mathrm{CN}), \\
732.43(\mathrm{C}-\mathrm{Cl})\end{array}$ & $\begin{array}{l}3.52\left(\mathrm{~s}, 2 \mathrm{H}, \mathrm{CH}_{2}\right), 7.64\left(\mathrm{~s}, 2 \mathrm{H}, \mathrm{NH}_{2}\right) \\
8.42(\mathrm{~s}, 1 \mathrm{H}, \mathrm{CH}), 7.54-7.63(\mathrm{~m}, 4 \mathrm{H}, \mathrm{Ar}- \\
\mathrm{H})\end{array}$ & $\left(\mathrm{M}^{+}\right) 284$ \\
\hline 5. & $2 \mathrm{e}$ & & $\begin{array}{l}1654.21(\mathrm{C}=\mathrm{N}), \\
1591.81(\mathrm{C}=\mathrm{C}), \\
3414.34 \text { and } 3285.67 \\
\left(\mathrm{NH}_{2}\right), 2249.76(\mathrm{CN}), \\
1447.93(\mathrm{C}-\mathrm{F})\end{array}$ & $\begin{array}{l}3.61\left(\mathrm{~s}, 2 \mathrm{H}, \mathrm{CH}_{2}\right), 7.73\left(\mathrm{~s}, 2 \mathrm{H}, \mathrm{NH}_{2}\right) \\
8.39(\mathrm{~s}, 1 \mathrm{H}, \mathrm{CH}), 7.57-7.64(\mathrm{~m}, 4 \mathrm{H}, \mathrm{Ar}- \\
\mathrm{H})\end{array}$ & $\left(\mathrm{M}^{+}\right) 268$ \\
\hline 6. & 3a & & $\begin{array}{l}1653.08(\mathrm{C}=\mathrm{N}), \\
1588.63(\mathrm{C}=\mathrm{C}), \\
3444.54 \text { and } 3281.32 \\
\left(\mathrm{NH}_{2}\right), 2243.76(\mathrm{CN})\end{array}$ & $\begin{array}{l}3.34\left(\mathrm{~s}, 2 \mathrm{H}, \mathrm{CH}_{2}\right), 7.78\left(\mathrm{~s}, 2 \mathrm{H}, \mathrm{NH}_{2}\right) \\
8.41(\mathrm{~s}, 1 \mathrm{H}, \mathrm{CH}), 7.55-7.59(\mathrm{~m}, 5 \mathrm{H}, \mathrm{Ar}- \\
\mathrm{H}), 11.52(\mathrm{~s}, 1 \mathrm{H}, \mathrm{OH})\end{array}$ & $\left(M^{+}\right) 298$ \\
\hline 7. & $3 b$ & & $\begin{array}{l}1687.24(\mathrm{C}=\mathrm{N}), \\
1589.11(\mathrm{C}=\mathrm{C}), \\
3451.65 \text { and } 3256.78 \\
\left(\mathrm{NH}_{2}\right), 2234.31(\mathrm{CN}), \\
778.98(\mathrm{C}-\mathrm{Cl})\end{array}$ & $\begin{array}{l}3.51\left(\mathrm{~s}, 2 \mathrm{H}, \mathrm{CH}_{2}\right), 7.62\left(\mathrm{~s}, 2 \mathrm{H}, \mathrm{NH}_{2}\right) \\
8.37(\mathrm{~s}, 1 \mathrm{H}, \mathrm{CH}), 7.52-7.58(\mathrm{~m}, 4 \mathrm{H}, \mathrm{Ar}- \\
\mathrm{H}), 11.23(\mathrm{~s}, 1 \mathrm{H}, \mathrm{OH})\end{array}$ & $\left(\mathrm{M}^{+}\right) 332$ \\
\hline 8. & $3 c$ & & $\begin{array}{l}1632.58(\mathrm{C}=\mathrm{N}), \\
1589.31(\mathrm{C}=\mathrm{C}), \\
3454.44 \text { and } 3268.91 \\
\left(\mathrm{NH}_{2}\right), 2247.04(\mathrm{CN}), \\
778.12(\mathrm{C}-\mathrm{Cl})\end{array}$ & $\begin{array}{l}3.11\left(\mathrm{~s}, 2 \mathrm{H}, \mathrm{CH}_{2}\right), 7.55\left(\mathrm{~s}, 2 \mathrm{H}, \mathrm{NH}_{2}\right) \\
8.39(\mathrm{~s}, 1 \mathrm{H}, \mathrm{CH}), 7.37-7.57(\mathrm{~m}, 4 \mathrm{H}, \mathrm{Ar}- \\
\mathrm{H}), 11.05(\mathrm{~s}, 1 \mathrm{H}, \mathrm{OH})\end{array}$ & $\left(\mathrm{M}^{+}\right) 332$ \\
\hline 9. & $3 d$ & & $\begin{array}{l}1651.32(\mathrm{C}=\mathrm{N}), \\
1598.12(\mathrm{C}=\mathrm{C}), \\
3464.07 \text { and } 3256.17 \\
\left(\mathrm{NH}_{2}\right), 2241.75(\mathrm{CN}), \\
756.76(\mathrm{C}-\mathrm{Cl})\end{array}$ & $\begin{array}{l}3.52\left(\mathrm{~s}, 2 \mathrm{H}, \mathrm{CH}_{2}\right), 7.77\left(\mathrm{~s}, 2 \mathrm{H}, \mathrm{NH}_{2}\right) \\
8.47(\mathrm{~s}, 1 \mathrm{H}, \mathrm{CH}), 7.50-7.56(\mathrm{~m}, 4 \mathrm{H}, \mathrm{Ar}- \\
\mathrm{H}), 11.41(\mathrm{~s}, 1 \mathrm{H}, \mathrm{OH})\end{array}$ & $\left(\mathrm{M}^{+}\right) 332$ \\
\hline 10. & $3 e$ & & $\begin{array}{l}1643.13(\mathrm{C}=\mathrm{N}), \\
1591.32(\mathrm{C}=\mathrm{C}), \\
3401.67 \text { and } 3239.31 \\
\left(\mathrm{NH}_{2}\right), 2208.89(\mathrm{CN}), \\
1432.76(\mathrm{C}-\mathrm{F})\end{array}$ & $\begin{array}{l}3.12\left(\mathrm{~s}, 2 \mathrm{H}, \mathrm{CH}_{2}\right), 7.67\left(\mathrm{~s}, 2 \mathrm{H}, \mathrm{NH}_{2}\right) \\
8.31(\mathrm{~s}, 1 \mathrm{H}, \mathrm{CH}), 7.48-7.60(\mathrm{~m}, 4 \mathrm{H}, \mathrm{Ar}- \\
\mathrm{H}), 11.41(\mathrm{~s}, 1 \mathrm{H}, \mathrm{OH})\end{array}$ & $\left(\mathrm{M}^{+}\right) 316$ \\
\hline
\end{tabular}




\section{Molecular docking}

The docking and binding free energy scores obtained from their respective receptor targets EGFR, ALK, VEGFR, and TNKS, confirmed the molecular interactions. The co-crystallised structures of gefitinib, ceritinib, axitinib, 3J1, which are active against lung cancer with the corresponding PDB IDs 4WKQ, 4Z55, 4AG8 and 4W5S, were obtained and found to have docking scores-8.80 kcal/mol,-11.36 $\mathrm{kcal} / \mathrm{mol},-14.41 \mathrm{kcal} / \mathrm{mol}$, and-13.95 $\mathrm{kcal} / \mathrm{mol}$ respectively; and their binding free energies are-95.15 kcal/mol,-100.94 kcal/mol,$123.86 \mathrm{kcal} / \mathrm{mol},-101.34 \mathrm{kcal} / \mathrm{mol}$ towards their respective receptors EGFR, ALK, VEGFR and TNKS, (table 1). The RMSD values of the crystallised structures showed RMSD values as $1.231 \AA$, 1.321
$\AA ̊, 1.412 \AA ̊$, $1.114 \AA$, respectively, which validated the docking results.

All the ten pyrazole derivatives screened for lung cancer targets exhibited docking values above- $5 \mathrm{kcal} / \mathrm{mol}$. The top pyrazole derivatives were 2e, 2d and 3d towards EGFR, ALK, VEGFR and TNKS. Their docking scores and binding affinity were given in table 2. In these top evaluations, $2 \mathrm{e}$ showed the best docking conformation with docking scores-7.75,-7.23,-8.52 and-8.31 $\mathrm{kcal} / \mathrm{mol}$ and binding free energy-62.77,-53.42,-77.78, and-63.67 $\mathrm{kcal} / \mathrm{mol}$ against, followed by pyrazoles $2 \mathrm{~d}(-7.70,-7.13,-8.47,-8.22$ $\mathrm{kcal} / \mathrm{mol})$ and $3 \mathrm{~d}(-7.51,-7.20,-8.30,-8.01 \mathrm{kcal} / \mathrm{mol}) \mathrm{EGFR}$, ALK, VEGFR and TNKS respectively (table 2).

Table 2: Structures, docking score and MMGBSA dG bind of reference compounds

\begin{tabular}{|c|c|c|c|c|}
\hline Reference compounds available in PDB & Structures & Target receptors & Docking scores & MMGBSA dG Bind \\
\hline Gefitinib & & 4WKQ & -8.806 & -95.15 \\
\hline Ceritinib & & $4 Z 55$ & -11.362 & -100.94 \\
\hline Axitinib & & 4AG8 & -14.414 & -123.86 \\
\hline 3J1 & & $4 \mathrm{~W} 5 \mathrm{~S}$ & -13.953 & -101.34 \\
\hline
\end{tabular}

To validate the chemical interactions, the analysis of co-crystals conformations are as follows; in enzyme EGFR, the common amino acids that make interactions with standard geftinib and pyrazole derivatives are Gln 791, Thr 790 and Thr 854 (hydrophobic); Met 793 (hydrogen bond); Leu 718, Leu 844, Met 793, Leu 792, Val 726, Ala 743, Met 766, Leu 788 (polar). Further, the common amino acids for the standard ceritinib and pyrazole derivatives that make interactions with enzyme ALK are Asn 1254; polar interactions are Leu 1122, Val 1130, Met 1199, Leu 1198, Leu 1256, Leu 1196, Val 1180, Ala 1148, Val 1180 and hydrogen bond with the same amino acid Met 1199. Similarly, for the enzyme, VEGFR, the common amino acids for axitinib and pyrazole derivatives that bond by hydrophobic interactions is Asn 923 and polar interactions are Cys 919, Phe 918, Val 916, Leu 1035, Ala 866, Val 899, Phe 1047, Cys 1045, Leu 840, Val 848. In the case of the TNKS enzyme, for the ligand 3J1 and pyrazoles, the amino acids that frequently make hydrophobic interactions are Ser 1221, Hid 1184, Ser 1186; Tyr 1224, Tyr 1213, Phe 1214, Ala 1215, Phe 1188, Pro 1187; polar interactions are Ala 1215, Phe 1214, Tyr 1213, Tyr 1203, Ile 1228, Pro 1187, Tyr 1224.

The finest docking conformations were also examined to reveal the primary interacting amino acid residues in the active pockets of EGFR, ALK, VEGFR and TNKS (table 3-5 and fig. 2-5).

Table 3: Docking score and MMGBSA dG bind of pyrazole derivatives

\begin{tabular}{|c|c|c|c|c|c|c|c|c|}
\hline \multirow[t]{2}{*}{ Compounds } & \multicolumn{4}{|c|}{ Docking scores (in kcal/mol) } & \multicolumn{4}{|c|}{ MMGBSA dG bind (in kcal/mol) } \\
\hline & $\begin{array}{l}\text { EGFR- } \\
\text { 4WKQ }\end{array}$ & $\begin{array}{l}\text { ALK- } \\
4 Z 55\end{array}$ & $\begin{array}{l}\text { VEGFR- } \\
4 \text { AG8 }\end{array}$ & TNKS-4W5S & $\begin{array}{l}\text { EGFR- } \\
\text { 4WKQ }\end{array}$ & ALK-4Z55 & $\begin{array}{l}\text { VEGFR- } \\
\text { 4AG8 }\end{array}$ & TNKS-4W5S \\
\hline $2 \mathrm{a}$ & -6.11 & -6.75 & -6.50 & -6.64 & -49.30 & -50.17 & -59.46 & -56.49 \\
\hline $2 \mathrm{~b}$ & -6.08 & -6.04 & -6.17 & -7.96 & -48.88 & -55.08 & -52.09 & -56.23 \\
\hline $2 c$ & -5.25 & -5.39 & -6.55 & -7.43 & -54.55 & -54.42 & -59.13 & -66.61 \\
\hline $2 d$ & -7.70 & -7.13 & -8.47 & -8.22 & -54.62 & -48.27 & -69.69 & -67.16 \\
\hline $2 \mathrm{e}$ & -7.75 & -7.23 & -8.52 & -8.31 & -62.77 & -53.42 & -77.78 & -63.67 \\
\hline $3 a$ & -6.97 & -7.04 & -7.83 & -7.06 & -61.04 & -53.37 & -68.07 & -58.26 \\
\hline $3 b$ & -6.35 & -5.91 & -7.72 & -7.02 & -60.63 & -57.81 & -85.72 & -69.41 \\
\hline $3 c$ & -7.44 & -7.06 & -7.66 & -7.94 & -65.99 & -59.02 & -81.08 & -79.86 \\
\hline $3 d$ & -7.51 & -7.20 & -8.30 & -8.01 & -63.06 & -55.07 & -78.91 & -73.87 \\
\hline $3 e$ & -7.21 & -6.75 & -7.48 & -7.91 & -60.18 & -50.9 & -70.68 & -64.4 \\
\hline
\end{tabular}


Table 4: Molecular interactions of reference compounds with the active site of protein

\begin{tabular}{|c|c|c|c|}
\hline $\begin{array}{l}\text { Reference } \\
\text { compounds }\end{array}$ & $\begin{array}{l}\text { Protein and } \\
\text { PDB IDs }\end{array}$ & Nature of interactions & Amino acids on active sites with \\
\hline \multirow[t]{6}{*}{ Gefitinib } & EGFR-4WKQ & Hydrophobic Interaction & Gln 791,Thr 790, Thr 854 \\
\hline & & Polar Interactions & $\begin{array}{l}\text { Leu 718, Leu 844, Met 793, Leu 792, Val 726, Ala 743, Met 766, Pro } \\
\text { 794, Phe 795, Leu } 788\end{array}$ \\
\hline & & H-Bond & Met 793, Cx 797 \\
\hline & & Halogen Bonding & Leu 788, Lys 745 \\
\hline & & Pi-Pi Stacking & -- \\
\hline & & Pi Cation & -- \\
\hline \multirow[t]{6}{*}{ Ceritinib } & ALK-4Z55 & Hydrophobic Interaction & Asn 1254 \\
\hline & & Polar Interactions & $\begin{array}{l}\text { Leu 1122, Val 1130, Met 1199, Leu 1198, Leu 1256, Leu 1196, Val } \\
\text { 1180, Ala 1148, Ala 1200, Val } 1180\end{array}$ \\
\hline & & H-Bond & Met 1199, Glu 1197, Lys 1150 \\
\hline & & Halogen Bonding & -- \\
\hline & & Pi-Pi Stacking & -- \\
\hline & & Pi Cation & -- \\
\hline \multirow[t]{6}{*}{ Axitinib } & VEGFR-4AG8 & Hydrophobic Interaction & -- \\
\hline & & Polar Interactions & $\begin{array}{l}\text { Phe 921, Cys 919, Phe 918, Leu 1035, Val 916, Ala 866, Val 899, Cys } \\
\text { 1045, Leu 840, Val 848, Phe 1047, Val 867, Val 914, Leu } 889\end{array}$ \\
\hline & & H-Bond & Asp 1046, Glu 885, Glu 917 \\
\hline & & Halogen Bonding & -- \\
\hline & & Pi-Pi Stacking & Phe 1047 \\
\hline & & Pi Cation & -- \\
\hline \multirow[t]{6}{*}{$3 \mathrm{~J} 1$} & TNKS 1-4W5S & Hydrophobic Interaction & Ser 1221, Hid 1184, Ser 1186, Hid 1201 \\
\hline & & Polar Interactions & $\begin{array}{l}\text { Tyr } 1203 \text {, Ile } 1228 \text {, Met } 1207, \text { Tyr } 1224 \text {, Tyr } 1213 \text {, Phe } 1214 \text {, Ala } \\
\text { 1215, Phe } 1188 \text {, Pro } 1187\end{array}$ \\
\hline & & H-Bond & Gly 1185, Glu 1291, Ser 1221 \\
\hline & & Halogen Bonding & -- \\
\hline & & Pi-Pi Stacking & Tyr 1224 \\
\hline & & Pi Cation & -- \\
\hline
\end{tabular}

Table 5: Molecular interactions of the pyrazole derivatives with the active site of protein

\begin{tabular}{|c|c|c|c|}
\hline Compounds & $\begin{array}{l}\text { Protein and } \\
\text { PDB IDs }\end{array}$ & Nature of Interactions & Amino acids on active sites \\
\hline \multirow[t]{14}{*}{$2 \mathrm{a}$} & EGFR-4WKQ & Hydrophobic Interaction & Gln 791, Thr 790, Thr 854 \\
\hline & & Polar Interactions & $\begin{array}{l}\text { Leu 718, Leu 844, Met 793, Leu 792, Val 726, Ala 743, Ile 744, Ile 789, Leu 788, } \\
\text { Leu 777, Met } 766\end{array}$ \\
\hline & & H-Bond & Met 793 \\
\hline & ALK-4Z55 & Hydrophobic Interaction & Hid 1124 \\
\hline & & Polar Interactions & $\begin{array}{l}\text { Leu 1122, Val 1130, Met 1199, Leu 1198, Leu 1256, Leu 1196, Val 1180, Ala } \\
\text { 1148, Val 1180, Ala } 1126\end{array}$ \\
\hline & & H-Bond & Met 1199 \\
\hline & VEGFR-4AG8 & Hydrophobic Interaction & Asn 923 \\
\hline & & Polar Interactions & $\begin{array}{l}\text { Cys 919, Phe 918, Val 916, Leu 1035, Ala 866, Val 899, Phe 1047, Cys 1045, Leu } \\
\text { 840, Val } 848\end{array}$ \\
\hline & & H-Bond & Cys 919 \\
\hline & & Pi-Pi Stacking & Phe 1047 \\
\hline & TNKS-4W5S & Hydrophobic Interaction & Ser 1221, Hid 1184, Ser 1186 \\
\hline & & Polar Interactions & Ala 1215 , Phe 1214 , Tyr 1213 , Met 1207 , Tyr 1203 , Ile 1228 , Pro 1187 , Tyr 1224 \\
\hline & & H-Bond & Tyr 1224 , Tyr 1203 \\
\hline & & Pi-Pi Stacking & Tyr 1224 \\
\hline \multirow[t]{15}{*}{$2 \mathrm{~b}$} & EGFR-4WKQ & Hydrophobic Interaction & Gln 791, Thr 790, Thr 854 \\
\hline & & Polar Interactions & $\begin{array}{l}\text { Leu 718, Leu 844, Met 793, Leu 792, Val 726, Ala 743, Ile 744, Ile 789, Leu 788, } \\
\text { Leu 777, Met } 766\end{array}$ \\
\hline & & H-Bond & Met 793 \\
\hline & & Halogen Bonding & Asp 855 \\
\hline & ALK-4Z55 & Hydrophobic Interaction & Hid 1124 \\
\hline & & Polar Interactions & $\begin{array}{l}\text { Leu 1122, Val 1130, Met 1199, Leu 1198, Leu 1256, Leu 1196, Val 1180, Ala } \\
\text { 1148, Val 1180, Ala } 1126\end{array}$ \\
\hline & & H-Bond & Lys 1150 , Ala 1126, Hid 1124 \\
\hline & & Halogen Bonding & Met 1199 \\
\hline & VEGFR-4AG8 & Hydrophobic Interaction & Asn 923 \\
\hline & & Polar Interactions & $\begin{array}{l}\text { Cys 919, Phe 918, Val 916, Leu 1035, Ala 866, Val 899, Phe 1047, Cys 1045, Leu } \\
\text { 840, Val } 848\end{array}$ \\
\hline & & H-Bond & Cys 919 \\
\hline & & Halogen Bonding & Glu 917 \\
\hline & TNKS-4W5S & Hydrophobic Interaction & Ser 1221, Hid 1184, Ser 1186, Hid 1201 \\
\hline & & Polar Interactions & $\begin{array}{l}\text { Ala } 1215 \text {, Phe } 1214, \text { Tyr } 1213 \text {, Tyr } 1203 \text {, Ile } 1228 \text {, Pro } 1187, \text { Tyr } 1224 \text {, Ala } 1202 \text {, } \\
\text { Phe } 1188 \text {, Phe } 1183\end{array}$ \\
\hline & & H-Bond & Tyr 1213, Hid 1201 \\
\hline
\end{tabular}




\begin{tabular}{|c|c|c|c|}
\hline Compounds & $\begin{array}{l}\text { Protein and } \\
\text { PDB IDs }\end{array}$ & Nature of Interactions & Amino acids on active sites \\
\hline & & Pi-Pi Stacking & Hid 1184, Tyr 1224 \\
\hline \multirow[t]{14}{*}{$2 c$} & EGFR-4WKQ & Hydrophobic Interaction & Gln 791, Thr 790, Thr 854 \\
\hline & & Polar Interactions & $\begin{array}{l}\text { Leu 718, Leu 844, Met 793, Leu 792, Val 726, Ala 743, Ile 744, Leu 788, Met 766, } \\
\text { Phe } 856\end{array}$ \\
\hline & & H-Bond & Met 793 \\
\hline & & Halogen Bonding & Leu 788, Lys 745, Ala 743 \\
\hline & ALK-4Z55 & Hydrophobic Interaction & Hid 1124 \\
\hline & & Polar Interactions & $\begin{array}{l}\text { Leu 1122, Val 1130, Met 1199, Leu 1198, Leu 1256, Leu 1196, Val 1180, Ala } \\
\text { 1148, Val } 1180\end{array}$ \\
\hline & & H-Bond & Glu 1197 \\
\hline & VEGFR-4AG8 & Polar Interactions & $\begin{array}{l}\text { Cys 919, Phe 918, Val 916, Leu 1035, Ala 866, Val 899, Phe 1047, Cys 1045, Leu } \\
\text { 840, Val 848, Val 914, Ile 915, Val 867, Leu 889, Val 898, Ile } 1044\end{array}$ \\
\hline & & Pi Cation & Lys 868 \\
\hline & TNKS-4W5S & Hydrophobic Interaction & Ser 1221, Hid 1184, Ser 1186, Hid 1201 \\
\hline & & Polar Interactions & $\begin{array}{l}\text { Phe } 1214 \text {, Tyr } 1213 \text {, Met } 1207 \text {, Tyr } 1203 \text {, Ile } 1228 \text {, Pro } 1187 \text {, Tyr } 1224 \text {, Ile } 1212 \text {, } \\
\text { Ala } 1202\end{array}$ \\
\hline & & H-Bond & Hid 1201 , Tyr 1213 \\
\hline & & Halogen Bonding & Ser 1221, Gly 1185 \\
\hline & & Pi-Pi Stacking & Tyr 1224, Hid 1184 \\
\hline \multirow[t]{16}{*}{$2 d$} & EGFR-4WKQ & Hydrophobic Interaction & Thr 790, Thr 854 \\
\hline & & Polar Interactions & Leu 718, Leu 844, Met 793, Leu 792, Val 726, Ala 743, Met 766, Pro 794 \\
\hline & & H-Bond & Csx 797, Met 793 \\
\hline & & Halogen Bonding & Cys 745 \\
\hline & ALK-4Z55 & Hydrophobic Interaction & Hid 1124 \\
\hline & & Polar Interactions & $\begin{array}{l}\text { Leu 1122, Val 1130, Met 1199, Leu 1198, Leu 1256, Leu 1196, Val 1180, Ala } \\
\text { 1148, Val } 1180\end{array}$ \\
\hline & & H-Bond & Glu 1197 \\
\hline & VEGFR-4AG8 & Hydrophobic Interaction & Asn 923, Thr 926 \\
\hline & & Polar Interactions & $\begin{array}{l}\text { Cys 919, Phe 918, Val 916, Leu 1035, Ala 866, Val 899, Phe 1047, Cys 1045, Leu } \\
840 \text {, Val } 848\end{array}$ \\
\hline & & H-Bond & Cys 919 \\
\hline & & Halogen Bonding & Asp 1046 \\
\hline & & Pi-Pi Stacking & Phe 1047 \\
\hline & TNKS-4W5S & Hydrophobic Interaction & Ser 1221, Hid 1184, Ser 1186 \\
\hline & & Polar Interactions & Ala 1215, Phe 1214, Tyr 1213, Met 1207, Tyr 1203, Ile 1228, Pro 1187, Tyr 1224 \\
\hline & & H-Bond & Tyr 1224, Tyr 1203 \\
\hline & & Pi-Pi Stacking & Tyr 1224 \\
\hline \multirow[t]{14}{*}{$2 \mathrm{e}$} & EGFR-4WKQ & Hydrophobic Interaction & Gln 791, Thr 790 \\
\hline & & Polar Interactions & Leu 718, Leu 844, Met 793, Leu 792, Val 726, Ala 743, Met 766, Pro 794 \\
\hline & & H-Bond & Csx 797, Met 793 \\
\hline & ALK-4Z55 & Hydrophobic Interaction & Hid 1124 \\
\hline & & Polar Interactions & $\begin{array}{l}\text { Leu 1122, Val 1130, Met 1199, Leu 1198, Leu 1256, Leu 1196, Val 1180, Ala } \\
\text { 1148, Val 1180, Ala } 1126\end{array}$ \\
\hline & & H-Bond & Ala 1126 , Lys 1150, Hid 1124 \\
\hline & VEGFR-4AG8 & Hydrophobic Interaction & Asn 923, Thr 926 \\
\hline & & Polar Interactions & $\begin{array}{l}\text { Cys 919, Phe 918, Val 916, Leu 1035, Ala 866, Val 899, Phe 1047, Cys 1045, Leu } \\
840, \text { Val } 848\end{array}$ \\
\hline & & H-Bond & Cys 919 \\
\hline & & Pi-Pi Stacking & Phe 1047 \\
\hline & TNKS-4W5S & Hydrophobic Interaction & Ser 1221, Hid 1184, Ser 1186, Hid 1201 \\
\hline & & Polar Interactions & $\begin{array}{l}\text { Ala } 1215 \text {, Phe } 1214, \text { Tyr } 1213 \text {, Tyr } 1203 \text {, Ile } 1228 \text {, Pro } 1187, \text { Tyr } 1224 \text {, Ala } 1202, \\
\text { Phe } 1188 \text {, Ile } 1212\end{array}$ \\
\hline & & H-Bond & Hid 1201 , Tyr 1213 \\
\hline & & Pi-Pi Stacking & Hid 1184, Tyr 1224 \\
\hline \multirow[t]{11}{*}{$3 \mathrm{a}$} & EGFR-4WKQ & Hydrophobic Interaction & Gln 791, Thr 790, Thr 854 \\
\hline & & Polar Interactions & $\begin{array}{l}\text { Leu 718, Leu 844, Met 793, Leu 792, Val 726, Ala 743, Met 766, Leu 788, Pro } \\
\text { 794, Cys } 775\end{array}$ \\
\hline & ALK-4Z55 & Hydrophobic Interaction & Ser 1206 \\
\hline & & Polar Interactions & $\begin{array}{l}\text { Leu 1122, Val 1130, Met 1199, Leu 1198, Leu 1256, Leu 1196, Val 1180, Ala } \\
\text { 1148, Val 1180, Ala } 1200\end{array}$ \\
\hline & VEGFR-4AG8 & Hydrophobic Interaction & Thr 926, Asn 923 \\
\hline & & Polar Interactions & $\begin{array}{l}\text { Phe 921, Cys 919, Phe 918, Leu 1035, Val 916, Ala 866, Val 899, Cys 1045, Leu } \\
\text { 840, Val 848, Phe } 1047\end{array}$ \\
\hline & & H-Bond & Cys 919, Leu 840 \\
\hline & TNKS-4W5S & Hydrophobic Interaction & Ser 1221, Hid 1184, Ser 1186, Hid 1201 \\
\hline & & Polar Interactions & $\begin{array}{l}\text { Ala } 1215 \text {, Tyr } 1213 \text {, Tyr } 1203 \text {, Ile } 1228 \text {, Pro } 1187, \text { Tyr } 1224 \text {, Phe } 1188 \text {, Ala } 1202 \text {, } \\
\text { Ile } 1212 \text {, Phe } 1214\end{array}$ \\
\hline & & H-Bond & Hid 1201, Tyr 1213 \\
\hline & & Pi-Pi Stacking & Hid 1184, Hid 1201 \\
\hline \multirow[t]{2}{*}{$3 b$} & EGFR-4WKQ & Hydrophobic Interaction & Gln 791, Thr 790, Thr 854 \\
\hline & & Polar Interactions & $\begin{array}{l}\text { Leu 718, Leu 844, Met 793, Leu 792, Val 726, Ala 743, Ile 789, Met 766, Leu 788, } \\
\text { Pro 794, Ile 744, Leu } 777\end{array}$ \\
\hline
\end{tabular}




\begin{tabular}{|c|c|c|c|}
\hline Compounds & $\begin{array}{l}\text { Protein and } \\
\text { PDB IDs }\end{array}$ & Nature of Interactions & Amino acids on active sites \\
\hline & \multirow{4}{*}{ ALK-4Z55 } & H-Bond & Met 793, Glu 791 \\
\hline & & Halogen Bonding & Lys 745 \\
\hline & & Hydrophobic Interaction & -- \\
\hline & & Polar Interactions & $\begin{array}{l}\text { Leu 1122, Met 1199, Leu 1198, Leu 1256, Leu 1196, Val 1180, Ala 1148, Val } \\
\text { 1180, Ala } 1200\end{array}$ \\
\hline & \multirow{3}{*}{ VEGFR-4AG8 } & H-Bond & Met 1199 \\
\hline & & Hydrophobic Interaction & Asn 923 \\
\hline & & Polar Interactions & $\begin{array}{l}\text { Phe 921, Cys 919, Phe 918, Leu 1035, Val 916, Ala 866, Val 899, Cys 1045, Leu } \\
\text { 840, Val 848, Phe 1047, Val 867, Val 914, Leu } 889\end{array}$ \\
\hline & \multirow{5}{*}{ TNKS-4W5S } & H-Bond & Cys 919 \\
\hline & & Pi-Pi Stacking & Phe 1047 \\
\hline & & Pi Cation & Lys 868 \\
\hline & & Hydrophobic Interaction & Ser 1221, Hid 1184, Ser 1186, Hid 1201 \\
\hline & & Polar Interactions & $\begin{array}{l}\text { Ala } 1215 \text {, Phe } 1214, \text { Tyr } 1213 \text {, Tyr } 1203 \text {, Ile } 1228 \text {, Pro } 1187, \text { Tyr } 1224 \text {, Ala } 1202 \text {, } \\
\text { Ile } 1212 \text {, Phe } 1188 \text {, Phe } 1183\end{array}$ \\
\hline & & Halogen Bonding & Tyr 1224, Tyr 1213 \\
\hline \multirow[t]{16}{*}{$3 c$} & \multirow{3}{*}{ EGFR-4WKQ } & Hydrophobic Interaction & Gln 791, Thr 790, Thr 854 \\
\hline & & Polar Interactions & Leu 718, Leu 844, Met 793, Leu 792, Val 726, Ala 743, Met 766, Leu 788, Pro 794 \\
\hline & & H-Bond & Met 793 \\
\hline & \multirow{3}{*}{ ALK-4Z55 } & Hydrophobic Interaction & Asn 1254 \\
\hline & & Polar Interactions & $\begin{array}{l}\text { Leu 1122, Val 1130, Met 1199, Leu 1198, Leu 1256, Leu 1196, Val 1180, Ala } \\
\text { 1148, Val 1180, Ala 1200, Ala } 1126\end{array}$ \\
\hline & & H-Bond & Lys 1150 , Met 1199 \\
\hline & \multirow[t]{5}{*}{ VEGFR-4AG8 } & Hydrophobic Interaction & Asn 923 \\
\hline & & Polar Interactions & $\begin{array}{l}\text { Phe 921, Cys 919, Phe 918, Leu 1035, Val 916, Ala 866, Val 899, Cys 1045, Leu } \\
\text { 840, Val 848, Phe 1047, Val 914, Leu } 889\end{array}$ \\
\hline & & H-Bond & Cys 919 \\
\hline & & Pi-Pi Stacking & Phe 1047 \\
\hline & & Pi Cation & Lys 868 \\
\hline & \multirow[t]{5}{*}{ TNKS-4W5S } & Hydrophobic Interaction & Ser 1221, Hid 1184, Ser 1186, Hid 1201 \\
\hline & & Polar Interactions & $\begin{array}{l}\text { Phe } 1214, \text { Tyr } 1213 \text {, Tyr } 1203 \text {, Ile } 1228 \text {, Pro } 1187 \text {, Tyr } 1224 \text {, Ile } 1212 \text {, Phe } 1188 \text {, } \\
\text { Phe } 1197 \text {, Ile } 1192\end{array}$ \\
\hline & & H-Bond & -- \\
\hline & & Halogen Bonding & Gly 1185 , Ser 1221 \\
\hline & & Pi-Pi Stacking & Tyr 1224 \\
\hline \multirow[t]{22}{*}{$3 d$} & \multirow[t]{6}{*}{ EGFR-4WKQ } & Hydrophobic Interaction & Thr 790, Thr 854 \\
\hline & & Polar Interactions & Leu 718, Leu 844, Met 793, Leu 792, Val 726, Ala 743, Met 766, Pro 794, Phe 795 \\
\hline & & H-Bond & Met 793 \\
\hline & & Halogen Bonding & Asp 855 \\
\hline & & Pi-Pi Stacking & -- \\
\hline & & Pi Cation & -- \\
\hline & \multirow[t]{6}{*}{ ALK-4Z55 } & Hydrophobic Interaction & Asn 1254 \\
\hline & & Polar Interactions & $\begin{array}{l}\text { Leu 1122, Val 1130, Met 1199, Leu 1198, Leu 1256, Leu 1196, Val 1180, Ala } \\
\text { 1148, Val 1180, Ala } 1200\end{array}$ \\
\hline & & H-Bond & Met 1199, Lys 1150 \\
\hline & & Halogen Bonding & -- \\
\hline & & Pi-Pi Stacking & -- \\
\hline & & Pi Cation & -- \\
\hline & \multirow[t]{6}{*}{ VEGFR-4AG8 } & Hydrophobic Interaction & Thr 926, Asn 923 \\
\hline & & Polar Interactions & $\begin{array}{l}\text { Phe 921, Cys 919, Phe 918, Leu 1035, Val 916, Ala 866, Val 899, Cys 1045, Leu } \\
\text { 840, Val 848, Phe } 1047\end{array}$ \\
\hline & & H-Bond & Cys 919, Leu 840 \\
\hline & & Halogen Bonding & Asp 1046 \\
\hline & & Pi-Pi Stacking & -- \\
\hline & & Pi Cation & -- \\
\hline & \multirow[t]{4}{*}{ TNKS-4W5S } & Hydrophobic Interaction & Ser 1221, Hid 1184, Ser 1186, Hid 1201 \\
\hline & & Polar Interactions & $\begin{array}{l}\text { Ala } 1215, \text { Phe } 1214, \text { Tyr } 1213 \text {, Tyr } 1203 \text {, Ile } 1228 \text {, Pro } 1187, \text { Tyr } 1224 \text {, Ile } 1212, \\
\text { Phe } 1188, \text { Phe } 1197 \text {, Ile } 1192 \text {, Ala } 1191\end{array}$ \\
\hline & & H-Bond & Ser 1186 \\
\hline & & Pi-Pi Stacking & Tyr 1224 \\
\hline \multirow[t]{11}{*}{$3 e$} & \multirow[t]{6}{*}{ EGFR-4WKQ } & Hydrophobic Interaction & Gln 791, Thr 790, Thr 854 \\
\hline & & Polar Interactions & Leu 718, Leu 844, Met 793, Leu 792, Val 726, Ala 743, Met 766, Leu 788, Phe 794 \\
\hline & & H-Bond & Met 793 \\
\hline & & Halogen Bonding & -- \\
\hline & & Pi-Pi Stacking & -- \\
\hline & & Pi Cation & -- \\
\hline & \multirow{5}{*}{ ALK-4Z55 } & Hydrophobic Interaction & Asn 1254 \\
\hline & & Polar Interactions & $\begin{array}{l}\text { Leu 1122, Val 1130, Met 1199, Leu 1198, Leu 1256, Leu 1196, Val 1180, Ala } \\
\text { 1148, Val 1180, Ala 1200, Ala } 1126\end{array}$ \\
\hline & & H-Bond & Met 1199 , Lys 1150 \\
\hline & & Halogen Bonding & -- \\
\hline & & Pi-Pi Stacking & -- \\
\hline
\end{tabular}




\begin{tabular}{|c|c|c|c|}
\hline Compounds & $\begin{array}{l}\text { Protein and } \\
\text { PDB IDs }\end{array}$ & Nature of Interactions & Amino acids on active sites \\
\hline & \multirow{7}{*}{ VEGFR-4AG8 } & Pi Cation & -- \\
\hline & & Hydrophobic Interaction & Asn 923 \\
\hline & & Polar Interactions & $\begin{array}{l}\text { Cys 919, Phe 918, Leu 1035, Val 916, Ala 866, Val 899, Cys 1045, Leu 840, Val } \\
848 \text {, Phe } 1047\end{array}$ \\
\hline & & H-Bond & Cys 919, Leu 840 \\
\hline & & Halogen Bonding & -- \\
\hline & & Pi-Pi Stacking & Phe 1047 \\
\hline & & Pi Cation & -- \\
\hline & \multirow[t]{6}{*}{ TNKS-4W5S } & Hydrophobic Interaction & Ser 1221, Hid 1184, Ser 1186, Hid 1201 \\
\hline & & Polar Interactions & $\begin{array}{l}\text { Ala } 1215 \text {, Phe } 1214 \text {, Tyr } 1213 \text {, Tyr } 1203 \text {, Ile } 1228 \text {, Pro } 1187 \text {, Tyr } 1224 \text {, Ile } 1212 \text {, } \\
\text { Phe } 1188 \text {, Phe } 1197 \text {, Ile 1192, Ala } 1191\end{array}$ \\
\hline & & H-Bond & Ser 1186 \\
\hline & & Halogen Bonding & -- \\
\hline & & Pi-Pi Stacking & Tyr 1224 \\
\hline & & Pi Cation & -- \\
\hline
\end{tabular}

a)

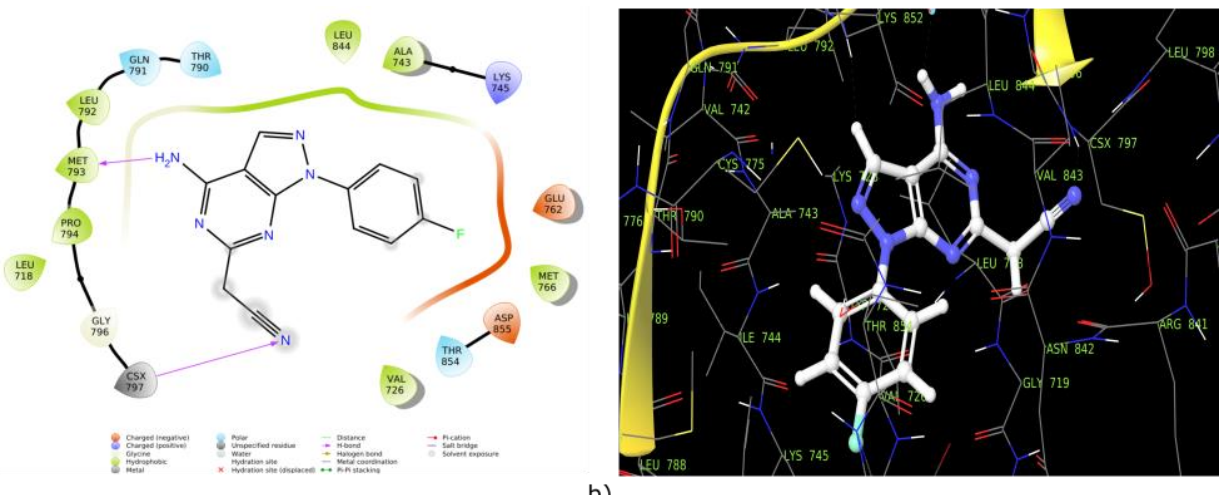

b)

Fig. 2: Molecular docking (a) 2D (b) 3D interactions of pyrazolopyrimidine 2e with 4 WKQ
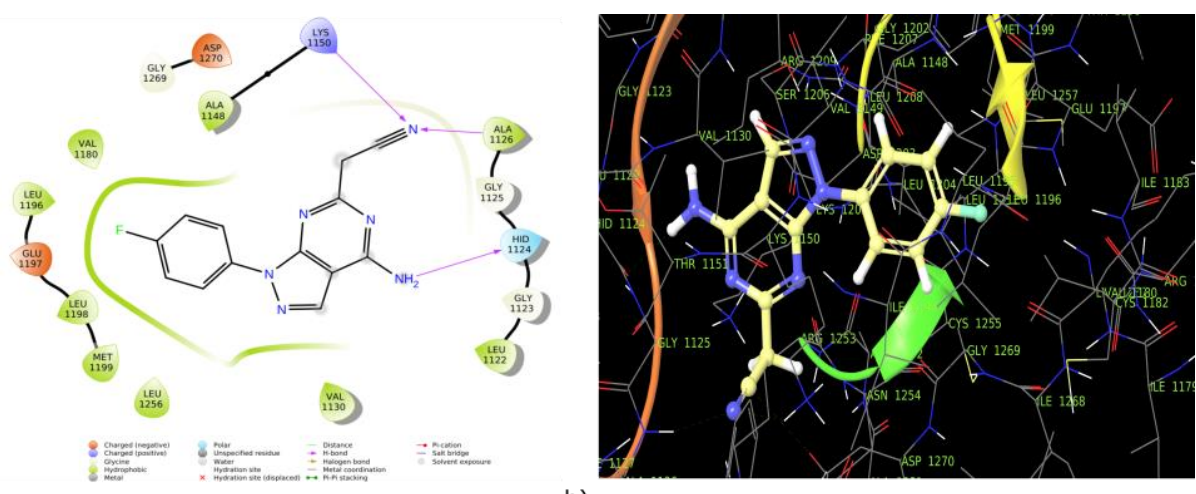

a)

b)

Fig. 3: Molecular docking (a) 2D (b) 3D interactions of pyrazolopyrimidine 2e with $4 \mathrm{Z55}$
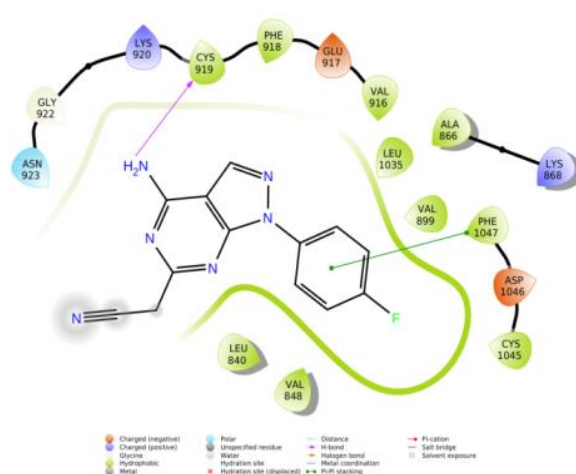

a)

Fig. 4: Molecular docking (a) 2D (b) 3D interactions of pyrazolopyrimidine 2e with 4AG8 

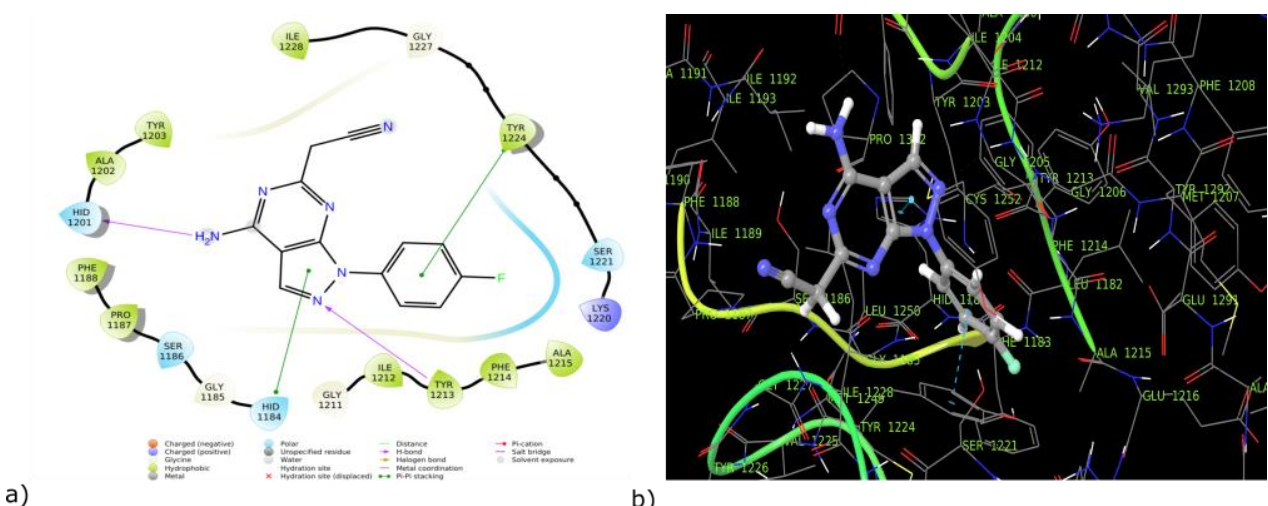

b)

Fig. 5: Molecular docking (a) 2D (b) 3D interactions of pyrazolopyrimidine 2e with 4W5S

\section{Pharmacophore hypothesis generation and modeling}

The results of all featured pharmacophore hypotheses are in table 6. DHRRR_1 is having the best survival score of 5.1979 in this study, which consists of one hydrophobic group $(\mathrm{H})$, one hydrogen bond donor (D), and three aromatic rings (R). The distances between the sites in the common pharmacophore hypothesis DHRRR_1 are given in fig. 6 (a-b) and table 7 .

Table 6: Score hypothesis

\begin{tabular}{|c|c|c|c|c|c|}
\hline Hypothesis ID & Survival score & Site score & Vector score & Volume & Selectivity \\
\hline DRRR_1 & 4.753541 & 0.967441 & 0.897639 & 0.778978 & 1.331332 \\
\hline DRRR_2 & 4.742687 & 0.966377 & 0.897544 & 0.777513 & 1.323101 \\
\hline ADRR_1 & 4.638511 & 0.966887 & 0.897754 & 0.779634 & 1.216084 \\
\hline ADRR_2 & 4.622626 & 0.970017 & 0.898106 & 0.777694 & 1.198658 \\
\hline ADRR_3 & 4.508706 & 0.942376 & 0.90864 & 0.690508 & 1.18903 \\
\hline DHRRR_1 & 5.197949 & 0.90054 & 0.861293 & 0.812639 & 2.021416 \\
\hline DHRRR_2 & 5.164714 & 0.882849 & 0.854915 & 0.809522 & 2.015368 \\
\hline ADHRR_1 & 5.000595 & 0.864909 & 0.861097 & 0.813654 & 1.858875 \\
\hline ADHRR_2 & 4.968628 & 0.864643 & 0.858113 & 0.807504 & 1.836308 \\
\hline ADHRR_3 & 4.915803 & 0.853138 & 0.86407 & 0.814323 & 1.782212 \\
\hline ADHRR_4 & 4.904614 & 0.868992 & 0.865852 & 0.813708 & 1.754002 \\
\hline ADHRR_5 & 4.898018 & 0.851548 & 0.85963 & 0.807438 & 1.777342 \\
\hline ADHRR_6 & 4.893939 & 0.846438 & 0.886796 & 0.781327 & 1.777319 \\
\hline ADHRR_7 & 4.881754 & 0.844744 & 0.859158 & 0.814788 & 1.761005 \\
\hline DHRR_5 & 4.529072 & 0.70509 & 0.955143 & 0.688846 & 1.481023 \\
\hline DHRR_1 & 4.712077 & 0.988826 & 0.852928 & 0.775829 & 1.492433 \\
\hline DHRR_2 & 4.684691 & 0.934964 & 0.862256 & 0.786716 & 1.498695 \\
\hline DHRR_3 & 4.682611 & 0.917492 & 0.865663 & 0.781164 & 1.51623 \\
\hline DHRR_4 & 4.620457 & 0.867853 & 0.852025 & 0.783564 & 1.514955 \\
\hline DHRRR_3 & 4.901226 & 0.663248 & 0.935309 & 0.690003 & 2.010606 \\
\hline
\end{tabular}

Table 7: Distances between different sites of model DHRRR_1

\begin{tabular}{|c|c|c|c|}
\hline S. No. & Site 1 & Site 2 & Distance \\
\hline 1. & H8 & D6 & 5.12 \\
\hline 2. & H8 & $\mathrm{R} 11$ & 3.16 \\
\hline 3. & H8 & R9 & 5.09 \\
\hline 4. & H8 & $\mathrm{R} 10$ & 6.53 \\
\hline 5. & D6 & $\mathrm{R} 11$ & 3.41 \\
\hline 6. & D6 & R9 & 4.57 \\
\hline 7. & D6 & R10 & 8.34 \\
\hline 8. & $\mathrm{R} 11$ & R9 & 2.15 \\
\hline 9. & $\mathrm{R} 11$ & $\mathrm{R} 10$ & 5.12 \\
\hline 10. & R11 & H8 & 3.16 \\
\hline 11. & $\mathrm{R} 11$ & D6 & 3.41 \\
\hline 12. & R9 & $\mathrm{R} 10$ & 3.97 \\
\hline 13. & R9 & H8 & 5.09 \\
\hline 14. & R9 & D6 & 4.57 \\
\hline 15. & R9 & $\mathrm{R} 11$ & 2.15 \\
\hline 16. & $\mathrm{R} 10$ & H8 & 6.53 \\
\hline 17. & $\mathrm{R} 10$ & D6 & 8.34 \\
\hline 18. & $\mathrm{R} 10$ & $\mathrm{R} 11$ & 5.12 \\
\hline 19. & $\mathrm{R} 10$ & $\mathrm{R} 9$ & 3.97 \\
\hline
\end{tabular}




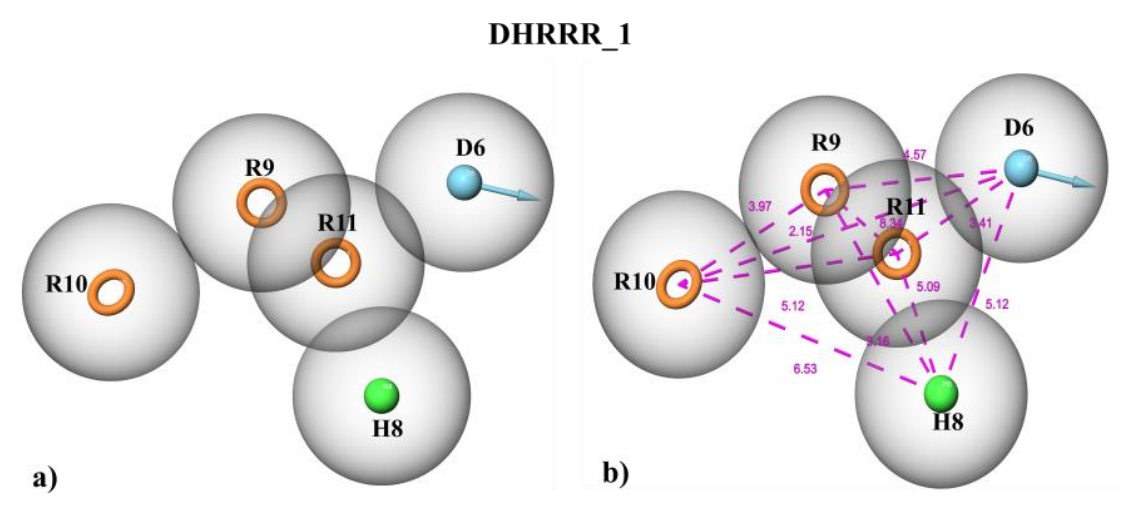

Fig. 6: a) Pharmacophore hypothesis DHRRR_1 b) Distances in the pharmacophore hypothesis DHRRR_1

Table 8: Physicochemical and ADMET properties of pyrazole derivatives

\begin{tabular}{|c|c|c|c|c|c|c|c|c|c|c|}
\hline S. No. & $\begin{array}{l}\text { Comp- } \\
\text { ounds }\end{array}$ & MW & $\log P$ & donorHB & $\begin{array}{l}\text { Accpt } \\
\text { HB }\end{array}$ & PSA & QPlogHERG & $\begin{array}{l}\text { QPP } \\
\text { Caco } \\
\end{array}$ & $\begin{array}{l}\text { QPlog } \\
\text { Khsa } \\
\end{array}$ & $\begin{array}{l}\text { Percent human oral } \\
\text { absorption }\end{array}$ \\
\hline 1. & Ceritinib & 577.743 & 4.838 & 2 & 9.75 & 119.604 & -7.51 & 54.854 & 1.146 & 73.44 \\
\hline 2. & Axitinib & 386.47 & 4.721 & 2 & 4.5 & 74.603 & -6.767 & 861.397 & 0.728 & 100 \\
\hline 3. & Gefitinib & 446.908 & 4.314 & 1 & 7.7 & 61.213 & -7.105 & 1044.67 & 0.351 & 100 \\
\hline 4. & $3 \mathrm{~J} 1$ & 332.361 & 2.438 & 2 & 6.7 & 89.242 & -6.288 & 352.29 & 0.097 & 86.808 \\
\hline 5. & $2 a$ & 250.262 & 1.41 & 2 & 5 & 89.799 & -5.273 & 236.605 & -0.209 & 77.689 \\
\hline 6. & $2 b$ & 284.707 & 1.859 & 2 & 5 & 90.018 & -5.241 & 237.627 & -0.107 & 80.355 \\
\hline 7. & $2 c$ & 284.707 & 1.897 & 2 & 5 & 89.767 & -5.223 & 236.907 & -0.108 & 80.553 \\
\hline 8. & $2 d$ & 284.707 & 1.895 & 2 & 5 & 89.789 & -5.208 & 236.913 & -0.109 & 80.543 \\
\hline 9. & $2 \mathrm{e}$ & 268.253 & 1.638 & 2 & 5 & 89.79 & -5.153 & 237.079 & -0.173 & 79.045 \\
\hline 10. & $3 a$ & 298.301 & 3.232 & 1 & 3 & 104.222 & -5.703 & 299.715 & 0.471 & 90.197 \\
\hline 11. & $3 b$ & 332.746 & 3.679 & 1 & 3 & 105.318 & -5.612 & 282.256 & 0.597 & 92.346 \\
\hline 12. & $3 c$ & 332.746 & 3.728 & 1 & 3 & 104.218 & -5.626 & 299.505 & 0.59 & 93.095 \\
\hline 13. & $3 d$ & 332.746 & 3.728 & 1 & 3 & 104.227 & -5.624 & 299.536 & 0.591 & 93.099 \\
\hline 14. & $3 e$ & 316.291 & 3.468 & 1 & 3 & 104.233 & -5.583 & 299.429 & 0.515 & 91.575 \\
\hline
\end{tabular}

Table 9: PASS prediction of anticancer properties

\begin{tabular}{|c|c|c|c|}
\hline & Compounds & Activity & $\mathbf{P a}$ \\
\hline \multirow[t]{5}{*}{1.} & $2 \mathrm{a}$ & Antineoplastic (melanoma) & 0.155 \\
\hline & & Antineoplastic antimetabolite & 0.108 \\
\hline & & Epidermal growth factor receptor kinase inhibitor & 0.142 \\
\hline & & ALK inhibitor & 0.107 \\
\hline & & Tankyrase inhibitor & 0.254 \\
\hline \multirow[t]{4}{*}{2.} & $2 \mathrm{~b}$ & ALK inhibitor & 0.101 \\
\hline & & Antineoplastic (melanoma) & 0.139 \\
\hline & & Tankyrase inhibitor & 0.174 \\
\hline & & Epidermal growth factor receptor kinase inhibitor & 0.133 \\
\hline \multirow[t]{3}{*}{3.} & $2 c$ & Epidermal growth factor receptor kinase inhibitor & 0.138 \\
\hline & & ALK inhibitor & 0.100 \\
\hline & & Tankyrase inhibitor & 0.182 \\
\hline \multirow[t]{3}{*}{4.} & $2 \mathrm{~d}$ & Tankyrase inhibitor & 0.192 \\
\hline & & Epidermal growth factor receptor kinase inhibitor & 0.146 \\
\hline & & ALK inhibitor & 0.108 \\
\hline \multirow[t]{3}{*}{5.} & $2 \mathrm{e}$ & Tankyrase inhibitor & 0.275 \\
\hline & & ALK inhibitor & 0.115 \\
\hline & & Epidermal growth factor receptor kinase inhibitor & 0.145 \\
\hline \multirow[t]{4}{*}{6.} & $3 a$ & Antineoplastic (melanoma) & 0.148 \\
\hline & & Antineoplastic antimetabolite & 0.113 \\
\hline & & ALK inhibitor & 0.097 \\
\hline & & Antileukemic & 0.205 \\
\hline \multirow[t]{3}{*}{7.} & $3 \mathrm{~b}$ & Antineoplastic (multiple myeloma) & 0.269 \\
\hline & & Antineoplastic (melanoma) & 0.136 \\
\hline & & ALK inhibitor & 0.094 \\
\hline \multirow[t]{2}{*}{8.} & $3 c$ & Antileukemic & 0.152 \\
\hline & & ALK inhibitor & 0.093 \\
\hline \multirow[t]{2}{*}{9.} & $3 d$ & Antineoplastic (multiple myeloma) & 0.223 \\
\hline & & ALK inhibitor & 0.098 \\
\hline \multirow[t]{4}{*}{10.} & $3 e$ & Antineoplastic antimetabolite & 0.102 \\
\hline & & ALK inhibitor & 0.104 \\
\hline & & Antileukemic & 0.186 \\
\hline & & Tankyrase inhibitor & 0.175 \\
\hline
\end{tabular}




\section{Drug-likeness, ADMET and prediction of activity spectral studies}

The synthesized ten pyrazoles have good drug-likeness properties, as shown in table 8 . We evaluated the physicochemical properties to fit into the Lipinski rule of five, which is a way to determine if they are orally bioavailable. The compounds have shown no violations for the Lipinski rule of 5. Their ADMET properties were analysed, and reported that all the compounds checked were found to have all the properties within the standard limit (table 8). The activity spectra for anticancer activity of the pyrazoles were predicted to find out the inhibitory effect on the particular enzymes (table 9). The compounds bearing pyrazolopyridines $(2 \mathrm{a}-2 \mathrm{e})$ are more effective against specific receptors such as EGFR, ALK and tankyrase.

\section{In vitro anticancer study by MTT assay}

The results of the cytotoxicity studies were presented in table 10 . Compound 2e, at the highest concentration, $200 \mu \mathrm{M}$, exhibited the most increased activity, which was $92 \%$ cytotoxic in nature and compounds $2 \mathrm{~d}$ and $3 \mathrm{~d}$ showed moderate cell growth inhibition around $80 \%$. On correlating with their docking scores, these compounds have excellently interacted with the four lung cancer targets. Thus the results interpret that the synthesized derivatives might inhibit any of the four targets discussed and exert their anticancer action. On further analysis of the top interacted pyrazole 2e, they have maximum interaction with the VEGFR receptor, which proves their mechanism.

Table 10: Cytotoxicity studies of the pyrazole derivatives

\begin{tabular}{|c|c|c|c|c|c|}
\hline \multirow[t]{3}{*}{ S. No. } & \multirow[t]{3}{*}{ Compound ID } & \multicolumn{4}{|c|}{ \% Cytotoxicity } \\
\hline & & \multicolumn{4}{|c|}{ Concentration $(\mu \mathrm{M})$} \\
\hline & & 25 & 50 & 100 & 200 \\
\hline 1. & $2 \mathrm{a}$ & 08 & 18 & 33 & 48 \\
\hline 2. & $2 b$ & 15 & 32 & 48 & 71 \\
\hline 3. & $2 c$ & 13 & 31 & 44 & 69 \\
\hline 4. & $2 \mathrm{~d}$ & 17 & 34 & 51 & 84 \\
\hline 5. & $2 \mathrm{e}$ & 40 & 58 & 78 & 92 \\
\hline 6. & $3 a$ & 09 & 20 & 30 & 45 \\
\hline 7. & $3 b$ & 17 & 34 & 51 & 78 \\
\hline 8. & $3 c$ & 08 & 19 & 36 & 53 \\
\hline 9. & $3 d$ & 11 & 26 & 51 & 85 \\
\hline 10. & $3 e$ & 10 & 22 & 38 & 56 \\
\hline
\end{tabular}

\section{DISCUSSION}

We found that the pyrazole condensed derivatives interacted with four lung cancer targets EGFR, ALK, VEGFR and TNKS, and their cytotoxicity action was proved against lung cancer. The compound 2e was the most active in both in silico and in vitro studies, followed by $3 \mathrm{~d}$ and $2 \mathrm{~d}$. Top compound $2 \mathrm{e}$ interacted with the VEGFR receptor excellently with stable binding mode and affinity. The best pharmacophore hypothesis, DHRRR_1 reveals the importance of the hydrogen bond donors, hydrophobic and aromatic groups essential for the anticancer action. Thus, validating the hydrogen bonds, hydrophobic groups and pi-pi interactions, which were showed by molecular docking. As per the cytotoxicity studies, the anticancer activity of the compounds $2 \mathrm{e}, 3 \mathrm{~d}$ and $2 \mathrm{~d}$ might be due to the introduction of electron-withdrawing fluorine and chlorine atoms in the benzene ring attached to the pyrazole ring.

Lung cancer development is stimulated by specific signaling pathways produced by receptors such as EGFR, ALK, VEGFR and TNKS. Much research has been performed to prove the anticancer efficacy of pyrazolopyrimidines on lung cancer [35], and some reported their inhibitory potentials on specific targets such as EGFR [36], VEGFR [37], tankyrase inhibitors [38] etc. We have screened the anticancer action by in vitro studies using A549 cell lines as a preliminary evaluation. Some reports are interfering in $\operatorname{EGFR~}[39,40]$ /VEGFR [41] /ALK [42] /Wnt [43, 44] /pathways inhibits the proliferation of A549 cell lines, and with this proof, we have carried the MTT assay. Cucurbitacin [39] and diazole [40] have been reported in proliferation inhibition in A549 cells by interfering EGFR signaling pathway. A study was performed to evaluate the TNKS small molecule inhibitor XAV939 on the proliferation and migration of lung adenocarcinoma A549 cells and found that XAV939 intervention inhibited A549 cell proliferation [43]. Determination of the appropriate target should be performed by analysing the enzyme antagonistic potential further, authenticating the mechanism of inhibition.

\section{CONCLUSION}

The synthesized pyrazole derivatives interacted well with the selected lung cancer targets-EGFR, ALK, VEGFR and TNKS; with their docking scores above- $5 \mathrm{kcal} / \mathrm{mol}$ equivalent with their standards. The molecular interactions are based on various parameters such as glide score, binding free energy, polar interactions, hydrophobic interactions, and hydrogen bond interactions. Further, the in vitro results exhibit compounds $2 \mathrm{e}$ as the best anti-lung cancer agents followed by $3 \mathrm{~d}$ and $2 \mathrm{~d}$, which was in agreement with their docking results. ADMET properties reported that all the compounds were found to have properties within the standard limit. The activity spectra of the pyrazoles predicted that pyrazolopyridines $(2 \mathrm{a}-2 \mathrm{e})$ are more effective against specific receptors such as EGFR, ALK and Tankyrase. Thus, this study suggests that the synthesized pyrazole derivatives can be further investigated to validate their enzyme inhibitory potentials by in vivo studies.

\section{ACKNOWLEDGEMENT}

We acknowledge Nitte Deemed to be University, Mangaluru, for the funding to carry out this project (University Research Grant No. NUFR1/2017/06/16). Also, thankful to the authorities of the NGSM Institute of Pharmaceutical Sciences, Mangaluru and NGSMIPS CADD lab for providing requirements for this work. Thanks to CUSAT, Cochin for NMR, Mysore University, Mysuru for Mass and Yenepoya Research Centre for anticancer studies.

\section{AUTHORS CONTRIBUTIONS}

All authors have contributed equally.

\section{CONFLICT OF INTERESTS}

The authors declare no conflict of interest, financial or otherwise.

\section{REFERENCES}

1. Prabhu VV, Devaraj N. Epidermal growth factor receptor tyrosine kinase: A potential target in the treatment of nonsmall-cell lung carcinoma.J Environ Pathol Toxicol Oncol. 2017;36(2):151-8. doi: 10.1615/JEnvironPatholToxicolOncol. 2017018341, PMID 2017018341.

2. Alferez D, Wilkinson RW, Watkins J, Poulsom R, Mandir N, Wedge SR, Pyrah IT, Smith NR, Jackson L, Ryan AJ, Goodlad RA. Dual inhibition of VEGFR and EGFR signaling reduces the incidence and size of intestinal adenomas in Apc(Min/+) mice. Mol Cancer Ther. 2008;7(3):590-8. doi: 10.1158/15357163.MCT-07-0433, PMID 18347145.

3. Antonicelli A, Cafarotti S, Indini A, Galli A, Russo A, Cesario A, Lococo FM, Russo P, Mainini AF, Bonifati LG, Nosotti M, Santambrogio L, Margaritora S, Granone PM, Dutly AE. EGFRtargeted therapy for non-small cell lung cancer: focus on EGFR 
oncogenic mutation. Int J Med Sci. 2013;10(3):320-30. doi: 10.7150/ijms.4609, PMID 23423768.

4. Castanon E, Martin P, Rolfo C, Fusco JP, Ceniceros L, Legaspi J, Santisteban M, Gil-Bazo I. Epidermal growth factor receptor targeting in non-small cell lung cancer: revisiting different strategies against the same target. Curr Drug Targets. 2014;15(14):1273-83.doi:

10.2174/138945011514141216092935, PMID 25511613.

5. Jänne PA, Yang JCH, Kim DW, Planchard D, Ohe Y, Ramalingam SS, Ahn MJ, Kim SW, Su WC, Horn L, Haggstrom D, Felip E, Kim JH, Frewer P, Cantarini M, Brown KH, Dickinson PA, Ghiorghiu S, Ranson M. AZD9291 in EGFR inhibitor-resistant non-smallcell lung cancer. N Engl J Med. 2015;372(18):1689-99. doi: 10.1056/NEJMoa1411817, PMID 25923549.

6. Gerber DE, Minna JD. ALK inhibition for non-small cell lung cancer: from discovery to therapy in record time. Cancer Cell. 2010;18(6):548-51. doi: 10.1016/j.ccr.2010.11.033, PMID 21156280.

7. Sang J, Acquaviva J, Friedland JC, Smith DL, Sequeira M, Zhang C, Jiang Q, Xue L, Lovly CM, Jimenez JP, Shaw AT, Doebele RC, He S, Bates RC, Camidge DR, Morris SW, El-Hariry I, Proia DA. Targeted inhibition of the molecular chaperone Hsp90 overcomes ALK inhibitor resistance in non-small cell lung cancer. Cancer Discovery. 2013;3(4):430-43. doi: 10.1158/2159-8290.CD-12-0440, PMID 23533265.

8. Nguyen-Ngoc $T$, Bouchaab $H$, Adjei AA, Peters S. BRAF alterations as therapeutic targets in non-small-cell lung cancer. J Thorac Oncol. 2015;10(10):1396-403. doi: 10.1097/JTO.0000000000000644, PMID 26301799.

9. Gautschi O, Milia J, Cabarrou B, Bluthgen MV, Besse B, Smit EF, Wolf J, Peters S, Früh M, Koeberle D, Oulkhouir Y, Schuler M, CurioniFontecedro A, Huret B, Kerjouan M, Michels S, Pall G, Rothschild S, Schmid-Bindert G, Scheffler M, Veillon R, Wannesson L, Diebold J, Zalcman G, Filleron T, Mazières J. Targeted therapy for patients with BRAF-mutant lung cancer: results from the European EURAF cohort. J Thorac Oncol. 2015;10(10):1451-7. doi: 10.1097/JTO.0000000000000625, PMID 26200454.

10. Feng Y, Hu J, Ma J, Feng K, Zhang X, Yang S, Wang W, Zhang J, Zhang Y. RNAi-mediated silencing of VEGF-C inhibits non-small cell lung cancer progression by simultaneously downregulating the CXCR4, CCR7, VEGFR-2 and VEGFR-3-dependent axes-induced ERK, p38 and AKT signalling pathways. Eur J Cancer. 2011;47(15):2353-63. doi: 10.1016/j.ejca.2011.05.006, PMID 21680174.

11. Villaruz LC, Socinski MA. The role of anti-angiogenesis in nonsmall-cell lung cancer: an update. Curr Oncol Rep. 2015;17(6):26. doi: 10.1007/s11912-015-0448-y, PMID 25947099.

12. Yang J, Chen J, He J, Li J, Shi J, Cho WC, Liu X. Wnt signaling as potential therapeutic target in lung cancer. Expert Opin Ther Targets. 2016;20(8):999-1015. doi: 10.1517/14728222. 2016.1154945, PMID 26882052.

13. Sigismund S, Avanzato D, Lanzetti L. Emerging functions of the EGFR in cancer. Mol Oncol. 2018;12(1):3-20. doi: 10.1002/1878-0261.12155, PMID 29124875.

14. Shackelford RE, Vora M, Mayhall K, Cotelingam J. ALKrearrangements and testing methods in non-small cell lung cancer: a review. Genes Cancer. 2014;5(1-2):1-14. doi: 10.18632/genes and cancer.3, PMID 24955213.

15. Alevizakos M, Kaltsas S, Syrigos KN. The VEGF pathway in lung cancer. Cancer Chemother Pharmacol. 2013;72(6):1169-81. doi: 10.1007/s00280-013-2298-3, PMID 24085262.

16. Riffell JL, Lord CJ, Ashworth A. Tankyrase-targeted therapeutics: expanding opportunities in the PARP family. Nat Rev Drug Discovery. 2012;11(12):923-36. doi: 10.1038/nrd3868, PMID 23197039.

17. Santarpia M, Liguori A, Karachaliou N, Gonzalez-Cao M, Daffina MG, D'Aveni A, Marabello G, Altavilla G, Rosell R. Osimertinib in the treatment of non-small-cell lung cancer: design, development and place in therapy. Lung Cancer (Auckl). 2017;8:109-25. doi: 10.2147/LCTT.S119644, PMID 28860885.

18. Descourt R, Perol M, Rousseau-Bussac G, Planchard D, Mennecier B, Wislez M, Cortot A, Guisier F, Galland L, Dô P, Schott R, Dansin E, Arrondeau J, Auliac JB, Chouaid C. Brigatinib in patients with ALKpositive advanced non-small-cell lung cancer pretreated with sequential ALK inhibitors: A multicentric real-world study (BRIGALK study).Lung Cancer. 2019;136:109-14. doi: 10.1016/j.lungcan.2019.08.010, PMID 31491676

19. Martinelli E, Troiani T, Morgillo F, Rodolico G, Vitagliano D, Morelli MP, Tuccillo C, Vecchione L, Capasso A, Orditura M, De Vita F, Eckhardt SG, Santoro M, Berrino L, Ciardiello F. Synergistic antitumor activity of sorafenib in combination with epidermal growth factor receptor inhibitors in colorectal and lung cancer cells. Clin Cancer Res. 2010;16(20):4990-5001. doi: 10.1158/1078-0432.CCR-10-0923, PMID 20810384.

20. Shukla P, Sharma A, Fageria L, Chowdhury R. Novel spiro/nonspiro pyranopyrazoles: eco-friendly synthesis, in vitro anticancer activity, DNA binding, and in-silico docking studies. Curr Bioact Compd. 2019;15(2):257-67. doi: $10.2174 / 1573407213666170828165512$.

21. Pj J, K IB. Synthesis, in silico physicochemical properties and biological activities of some pyrazoline derivatives. Asian J Pharm Clin Res. 2017;10(4):456-9. doi: 10.22159/ ajpcr.2017.v10i4.17093.

22. Bhat IS, Jainey PJ. Antimicrobial studies of some substituted pyrazoline derivatives derived from acetyl hydrazines. Asian J Pharm Clin Res. 2014;7(4):237-9.

23. James JP, Kumar P, Kumar A, Bhat KI, Shastry CS. In silico anticancer evaluation, molecular docking and pharmacophore modeling of flavonoids against various cancer targets. Lett Drug Des Discovery. 2020 Dec 1;17(12):1485-501. doi: 10.2174/1570180817999200730164222.

24. Kodical DD, James JP, Deepthi K, Kumar P, Cyriac C, Gopika KV. ADMET, Molecular docking studies and binding energy calculations of pyrimidine-2-thiol derivatives as cox inhibitors. Res J Pharm Technol. 2020 Sep 1;13(9):4200-6. doi: 10.5958/0974-360X.2020.00742.8.

25. Salaheldin AM, Oliveira Campos AMF, Rodrigues LM. Heterocyclic synthesis with nitriles: synthesis of pyrazolopyrimidine and pyrazolopyridine derivatives. Synth Commun. 2009;39(7):1186-95. doi: $10.1080 / 00397910802517814$.

26. Schrödinger. Schrödinger Release; 2019. Vol. 4. Available from: https://www.schrodinger.com. [Last accessed on 10 Jul 2021]

27. PDB database. Available from: https://www.rcsb.org/ structure/4WKQ. [Last accessed on 10 Jul 2021]

28. Michellys PY, Chen B, Jiang T, Jin Y, Lu W, Marsilje TH, Pei W, Uno T, Zhu X, Wu B, Nguyen TN, Bursulaya B, Lee C, Li N, Kim S, Tuntland T, Liu B, Sun F, Steffy A, Hood T. Design and synthesis of novel selective anaplastic lymphoma kinase inhibitors. Bioorg Med Chem Lett. 2016;26(3):1090-6. doi: 10.1016/j.bmcl.2015.11.049, PMID 26750252.

29. McTigue M, Murray BW, Chen JH, Deng YL, Solowiej J, Kania RS. Molecular conformations, interactions, and properties associated with drug efficiency and clinical performance among VEGFR TK inhibitors. Proc Natl Acad Sci USA 2012;109(45):18281-9. doi: 10.1073/pnas.1207759109, PMID 22988103.

30. Johannes JW, Almeida L, Barlaam B, Boriack Sjodin PA, Casella RP, Croft RA, Dishington AP, Gingipalli L, Gu C, Hawkins JL, Holmes JL, Howard T, Huang J, Ioannidis S, Kazmirski S, Lamb ML, McGuire TM, Moore JE, Ogg D, Patel A, Pike KG, Pontz T, Robb GR, Su N, Wang H, Wu X, Zhang HJ, Zhang Y, Zheng X, Wang T. Pyrimidinone nicotinamide mimetics as selective tankyrase and wnt pathway inhibitors suitable for in vivo pharmacology. ACS Med Chem Lett. 2015;6(3):254-9. doi: 10.1021/ml5003663, PMID 25815142.

31. Friesner RA, Murphy RB, Repasky MP, Frye LL, Greenwood JR, Halgren TA, Sanschagrin PC, Mainz DT. Extra precision glide: docking and scoring incorporating a model of hydrophobic enclosure for protein-ligand complexes. J Med Chem. 2006;49(21):6177-96. doi: 10.1021/jm051256o, PMID 17034125.

32. Lipinski CA, Lombardo F, Dominy BW, Feeney PJ. Experimental and computational approaches to estimate solubility and permeability in drug discovery and development settings. Adv Drug Delivery Rev. 2001;46(1-3):3-26. doi: 10.1016/s0169409x(00)00129-0, PMID 11259830.

33. Druzhilovskiy DS, Rudik AV, Filimonov DA, Lagunin AA, Gloriozova $\mathrm{TA}$, Poroikov VV. Online resources for the prediction of biological 
activity of organic compounds. Russ Chem Bull. 2016;65(2):38493. doi: 10.1007/s11172-016-1310-6.

34. Denizot F, Lang R. Rapid colorimetric assay for cell growth and survival. J Immunol Method. 1986;89(2):271-7. doi: 10.1016/0022-1759(86)90368-6.

35. El-Kalyoubi SA. Synthesis and anticancer evaluation of some novel pyrimido[5,4-e] $[1,2,4]$ triazines and pyrazolo[3,4d]pyrimidine using DMF-DMA as methylating and cyclizing agent. Chem Cent J. 2018;12(1):64. doi: 10.1186/s13065-0180424-3, PMID 29796716.

36. Ismail NSM, Ali EMH, Ibrahim DA, Serya RAT, Abou El Ella DA. Pyrazolo [3, 4-d] pyrimidine-based scaffold derivatives targeting kinases as anticancer agents. Future J Pharm Sci. 2016;2(1):20-30. doi: 10.1016/j.fjps.2016.02.002.

37. Sun N, Ji H, Wang W, Zhu Q, Cao M, Zang Q. Inhibitory effect of dexamethasone on residual Lewis lung cancer cells in mice following palliative surgery. Oncol Lett. 2017;13(1):356-62. doi: 10.3892/ol.2016.5422, PMID 28123567.

38. Chedid M, Eissa HO, Engler TA, Furness KW, Woods TA, Wrobleski AD. US Patent No. 9,624,218. Washington, DC: US Patent and Trademark Office; 2017.

39. Zhang J, Song Y, Liang Y, Zou H, Zuo P, Yan M, Jing S, Li T, Wang Y, Li D, Zhang T, Wei Z. Cucurbitacin IIa interferes with EGFRMAPK signaling pathway leads to proliferation inhibition in A549 cells. Food Chem Toxicol. 2019 Oct 1;132:110654. doi: 10.1016/j.fct.2019.110654.
40. Vinod Prabhu V, Elangovan P, Niranjali Devaraj S, Sakthivel KM. Targeting apoptosis by 1, 2-diazole through regulation of EGFR, Bcl-2 and CDK-2 mediated signaling pathway in human nonsmall cell lung carcinoma A549 cells. Gene. 2018 Dec 30;679:352-9. doi: 10.1016/j.gene.2018.09.014, PMID 30218747.

41. Shi L, Zhang S, Wu H, Zhang L, Dai X, Hu J, Xue J, Liu T, Liang Y, Wu G. MiR-200c increases the radiosensitivity of non-small-cell lung cancer cell line A549 by targeting the VEGF-VEGFR2 pathway. PLOS ONE. 2013 Oct 30;8(10):e78344. doi: 10.1371/journal.pone.0078344, PMID 24205206.

42. Yang L, Li G, Zhao L, Pan F, Qiang J, Han S. Blocking the PI3K pathway enhances the efficacy of ALK-targeted therapy in EML4-ALK-positive non-small-cell lung cancer. Tumour Biol. 2014 Oct;35(10):9759-67. doi: 10.1007/s13277-014-2252-y, PMID 24972969.

43. Li C, Zheng X, Han Y, Lv Y, Lan F, Zhao J. XAV939 inhibits the proliferation and migration of lung adenocarcinoma A549 cells through the WNT pathway. Oncol Lett. 2018 Jun 1;15(6):8973-82. doi: 10.3892/ol.2018.8491, PMID 29805633

44. Li P, Zhao S, Hu Y. SFRP2 modulates non-small cell lung cancer A549 cell apoptosis and metastasis by regulating mitochondrial fission via Wnt pathways. Mol Med Rep. 2019 Aug 1;20(2):1925-32. doi: 10.3892/mmr.2019.10393, PMID 31257495 . 